\title{
Theory of the Whole and the part - Ontological Perspective (E. Husserl, R. Ingarden)
}

\author{
Katarzyna Barska \\ Jagiellonian University, Poland \\ e-mail: barska.katarzyna@gmail.com
}

\begin{abstract}
:
The purpose of the paper is demonstrate the thesis that Ingarden's ontological system allows a better understanding of the "part-whole" problem then previous theories. Especially, if we take into account the existential ontology of Ingarden, which refers to Husserl "part-whole" theory, we can see that development of terms made by Ingarden sheds new light on old problems. In this context, particularly important is to distinguish between two existential moments: contingancy/inseparatness, because thanks to them we can talk about many different types of relationships and hence many types of objects.

Keywords: existential moments, part, whole, object, Husserl, Ingarden.
\end{abstract}

\section{Introduction to the topic}

To honestly discuss moments of existential separateness and inseparateness, it will be necessary to elaborate previous contexts of understanding for these terms. This task, however, appears to be very difficult for, at least, a few reasons ${ }^{1}$. Terminological confusion around the terms: 'inseparate' [ $\mathrm{x}$ ' is inseparate if it can exist only together with certain other ' $y$ ' in the unity of a whole, alternatively 'non-self-sufficient'], 'incontingent', 'heteronomous', 'inderivate' and their oppositions is the first, and, at the same time, the greatest difficulty.

Therefore, my objective here will be to clarify these terms and the way they are understood according to the Ingarden's idea and indicate misunderstandings, which may be encountered in the literature on this subject as a result of this confusion.

In many instances, it will allow to refute the arguments against locating moments of separateness and inseparateness in the existential area. At the same time, it will turn out that the existential moments are not merely relationships between objects and they may not be reduced to them, and above all, what is my greatest concern, it will be demonstrable that ignoring the distinction inseparate-incontingent, and thus choosing the option with poorer ontology leads to giving up the solutions that are essential from ontological point of view ${ }^{2}$.

In short, my intention is to demonstrate that Ingarden's existential classification is relatively the best tool for developing ontology as such. This is because it allows you to refer to multiple types of objects, which in turn is an exemplification of adequacy of the existential and formal ontology. This may not be achieved when using the term 'contingency' [the dependence of a separate entity on another entity in order to remain in existence, alternatively 'existential dependence'] that would (without distinguishing) comprise those types of moments, regardless of whether these studies would ultimately be existential or formal studies. 
This valuation is significant, especially when we take into account Ingarden's suggestion concerning the role of ontology in formulating metaphysical systems.

My intention is also to make foundations for the Ingarden's hypothesis that understanding the moment of existential inseparateness goes beyond both the formal and existential area. The proper understanding of this moment is rooted in the material categories. More precisely speaking, this is, of course, the existential moment with qualitative reference of object ${ }^{3}$. It will also be necessary to determine precisely understanding of the formal categories which existential moments undoubtedly involve, i.e.: an object, the whole-the part, taking into account all divisions they include (an object-something, an object as composition of matter, form and mode of being, an object as the subject of properties, the whole as a unity, the whole as a summative whole, as well as the autonomous and heteronomous object).

As shown in the above example, even having initially arranged accents, they are distributed differently, from the material, through the formal, ending with the existential ones. This is because considerations in the field of formal, existential and material ontology intersect.

It turns out for instance that two basic subtypes of inseparateness distinguished by Ingarden from among many of them, i.e. material and formal inseparateness are not of the same rank. Due to being distinguished in material terms, the inseparateness allows to overcome regarding separateness-inseparateness opposition as a formal and ontological opposition. It is commonly accepted that this was Husserl's position and perhaps it is not unreasonable that it is imputed to Ingarden as well, and thus this part of his existential considerations is being degraded.

Husserl begins analysing the separate and inseparate content, starting with the Stumpf's findings and then he refers them respectively to the specific and abstract content. Particular attention should be paid to how this reference is understood. Taking into account the perspective of Stumpf's analyses, i.e. considering the inseparate and separate content as the contents of consciousness, it must be added that the Husserlian term 'content' is not limited to the phenomenological content.

Similarly, in the initial analyses, the terms 'object' ('content'), and 'part' are understood very broadly. Therefore, foundations for the difference between the concrete and the abstract may not be found in - in words of Husserl - phenomenological facts, because they belong to the realm of acts which means that, in the first instance, the concrete is given directly (separate) in the presentation, while in the second instance, what is perceived is the abstract content as inseparate and requiring being attached to the presentation of the relevant concrete this content is founded on.

The separate-inseparate opposition being differentiated within this particular realm is not the case either:

We've come far enough to see that when considering the difference between distinctive and indistinctive content (or, if you prefer [to use a different name], between the content presentable and unpresentable for themselves, separate and inseparate - since these expressions seem to be obvious here) we move within the realm of inexact ['subjective' intuition, having peculiar essential properties as well], and that applying this difference we will never reach to [the general ontological difference] between abstract and concrete content, or, as we decided to call them above, separate and inseparate [content] [4, p. 326].

The criterion to distinguish the separate and inseparate content founded on different acts: presentation for itself and noticing for itself, is also challenged by Husserl. Expressions 'for itself' in both acts operate in identical manner, thus they blur the difference between them. If we agree that the expressions 'for itself' in presentation is to be equivalent in meaning to the term 'separate', then it operates in terms of logic. In this case, the act of presentation may be interchangeable with the act of thinking and in this sense it refers to objective states of affairs, and therefore ontological 
laws rather than subjective perceptions. The difference between a separate and an inseparate object (content) is founded in the thing itself or in essence of that thing, rather than in the way it is perceived: 'What we can not think about may not exist, what can not exist, we can not think about this equivalence determines the difference between the very concept of thinking and presentation to oneself and thinking in the regular and subjective sense' [4, p. 293].

This is a very clear evidence that analyses of what is separate and inseparate, have been moved into the ontological dimension.

All previous meanings of abstraction may not be a foundation to identify the abstract and concrete content with what is inseparate and separate. Both abstraction - as an act of distinction in the intuitive the abstract content presentation, also perceived intuitively - and abstraction, which itself is not an act, but a property of the phenomenal part of an act consisting in some kind of highlighting, stressing the abstract content, still not perceived intuitively, but being a carrier of a specific intention (in phenomenological reflection); and also abstracting as a peculiar act of extracting the abstract content from the background - may not be a foundation to distinguish what is separate from what is inseparate, although what is inseparate and what is separate can be given as part of the three elements mentioned above. It is not out of the question however that, in this sense, we can abstract separate "objects".

Similarly, the inseparate content may be perceived intuitively, therefore they are 'parts' of objects. So it is not the case as distinctio rationis teaching states, i.e. that objects have only separate parts - pieces that can be detached from them and presented separately. On the other hand, the inseparate content identified with abstract ideas (Locke), as general meanings, are detached from specific intuitive acts. It is also not the case that - according to Husserl - highlighting and emphasising abstract content is equivalent to formation of abstract concepts.

Therefore, the act of noticing the abstract content is one thing, and the act of general presentation is quite another thing. In the latter, intention is directed to species; specific intuitive acts are foundations for the intention that is fulfilled, emphasising the abstract content, it is not them however, but species what is intended. These acts are not abstract content either.

As these critical studies imply, inseparate or abstract moments, which make up an object, are still confused with species, respective, subjectively experienced abstract content is confused with abstract concepts (meanings of certain names) while [emphases, resp.] acts of noticing this abstract content are confused with acts of general presentation [4, p. 266].

Transferring the difference between what is inseparate and what is separate within the realm of acts of presentation or phenomenological content, at the same time confusing the immanent object with the semantic content of a presentation, results in confusing the abstract content within an object, with species. Husserl points out that it is this position that leads to refusing to adopt the thesis on the intuitive nature of the abstract content. He refers to positions of Höfler and Meinong: 'The intuitive nature of the abstract content is denied in the same way, although, as moments of concrete intuition, they are perceived intuitively as well; this is true, since what deceives us here is [sensual] unintuitiveness of general concepts'[4, p. 267].

Therefore, in the field of ontology, what exists are individual and general (specific) objects. Adopting this thesis allows to avoid earlier equivocation, in which general objects and general meanings (general presentations) are called concepts. This equivocation has led to next equivocation of name and object of presentation and its content (the meaning of the name). This kind of confusion is a result, as it seems, of different acts of individual and general presentation of object. Presentation of the latter is founded on the presentation of the concrete, whether conceived as a whole or as its parts (property) perceived as highlighted part of the act as well, which underlies the separate act (separate founded act). 
No part or property of presentation of an individual, mentioned above, contains species. Therefore, presumption of the property of an object is not presumption of species. While attempting to grasp the content of an object, a new method of presentation is constructed under which species can be referred to. To put it simply, we can say that when species is the object of presentation, it is given via mediation of an individual object and its properties, so in this perspective, also relationships between species and an individual object are given. It is acceptable to recognise that what is inseparate - abstract in the ontological perspective of individual (concretes) and general (specific) objects - is equivalent to a part of an object, if it itself is not an object or a concrete. The latter condition is only in as much important as certain continuity may happen, even inseparable (in the presentation), but referred to the separate parts, because they would be concretes e.g. parts of equally coloured surface.

For Husserl, (in)separateness is grounded in the material beings, in the essence of the content. In terms of epistemology, it corresponds to synthetic laws a priori. Therefore, it is not an analytical necessity, because it is a consequence of analytical laws which contain only formal concepts and the corresponding theorems are formulated without peculiarity of content of objects. They may be totally formalised, because they are not sensitive to any material aspect. Matter can always be replaced with a pure form.

The process of abstraction may be understood as disregarding matter totally (formalisation) while ignoring recognising the existence of individuals and the all relationships of empirical nature, which is expressed by their mere being a priori. Empirical relationships are ignored in similar manner when considering synthetic laws a priori, this time however, abstraction is of ideating nature (generalisation) and to put it simply, it is quite the opposite, because necessity is derived from matter, and abstracts from form ${ }^{4}$.

At this point, another difficulty arises, which means that objectivity (universality and necessity) of laws derived based on relationships between material entities may be questioned. After all, it is acceptable to assume that necessities we mentioned before, are grounded in empirical relationships or sensory impressions, which means that they are always grasped as based on of sensory data.

The first option forces us to abandon a priori laws by returning to actuality, the second option forces us to return to subjectivity. This is not a sufficient premise to undermine the position presented, because the derivation of necessity of this particular ground is not sufficient to give rise to further reductionism.

I will not get more into detail with this issue, because solving it is not yet necessary to analyse the issues being taken, and the nature of the problem would require extensive research in epistemology, which in the final context - in the context of non-reductionist Ingarden's ontology we adopted here - are not necessary. But let me just quote, as it seems, a similar position to issues we outlined, taken by Piotr Łaciak in his article 'Kant and Husserl and the Problem of the Material a priori':

In view of considerations set out in this text, it becomes obvious that the dependency on sensory impressions does not have to be evidence of the empirical cognition, for in view of both Kant and Husserl, non-pure cognition a priori as non-pure is dependent on sensory impressions because it refers to the material content of things, and at the same time as a priori, it is independent from experience, because it meets the requirement of universality and necessity [7, p. 55] (see also [8]).

What is most important and worth emphasising can be generalised so that all sources of inseparateness refer to the material ontology. Necessity of coexistence of inseparate moments is implied by material relationships of essence, not from the formal categories. Husserl expresses it explicitly: 
No one will regard laws such as the law of causality that describes inseparateness of changes [in the real objects] or (usually the way they are formulated is not satisfactory) laws that describe inseparateness of pure qualities, intensities, extensions, limits, forms, relationships, etc., as equal to [pure 'analytical'] general laws, such as the whole can not exist without parts, or [analytical necessities such as] a king, a lord, a father can not exist without lieges, servants, children, etc. In general we can say here: correlates demand one another, they can not be conceived without one another, resp. they can not exist. If we compare this with any specific theorem from the opposite group, such as a colour can not exist without something that is coloured, or a colour can not exist without a certain extension it coats - the difference is blatantly obvious immediately. Colour is not a relational expression, whose meaning would contain in itself a reference to something else. Although colour 'can not be conceived' without a coloured object, after all existence of is a coloured object and more precisely: extension, is not 'analytically' grounded in the concept of colour [4, pt. I, p. 311-312].

\section{Eugenia Ginsberg's Criticism of Husserl's Theory of the Whole and the Part}

When classifying the moment of inseparateness as an existential moment, Ingarden distinguishes formal and material types of existential inseparateness, the first one however is subordinate to the other. This reference is important, especially when considering the Ginsberg's counter arguments put forward to Husserl's statements. The thesis on whether division into separate and inseparate parts corresponds to identical division in the area of objects is questioned. The term 'object' is understood extremely broadly, it is interchangeable with the term 'something'. This is because it seems unlikely that all objects are parts of some kind of the whole.

Husserl's position would be more understandable if Ingarden's meaning was used already at this point of the analysis. If we assume that Husserl discusses both (in)separateness as well as contingencies, then, indeed, the Absolute would be the only independent object. Obviously, in Ingarden's perspective, what is contingent, may be a part of some other thing (always separate), but does not have to. There is a discrepancy between Husserl and Ingarden at this point. Later in this discussion, we will find to what extent these differences are merely of terminological nature.

Returning to the objections raised by Ginsberg and, at the same time, maintaining Husserlian assumptions, i.e. the term 'object' being conceived in the most general meaning, while the word 'part' means only real parts, what is disputed in determining inseparateness are ${ }^{5}$ :

a) necessity of existence of an object complementary to this object and

b) existence of a heteronomous object as part of the whole, which is created along with objects complementary to it.

Prior to examining in detail Ginsberg's examples that question the Husserlian implications, which are inherent to describe inseparateness, let me point out that some kind of confusion becomes apparent even in what has already been quoted above. Hence the call for proper understanding of the terms 'object' and 'part'. The first term is clear, as used by Twardowski and Meinong, it is the most general concept of object, interchangeable with the term 'something', therefore, in this interpretation, each part is an object. This in no way implies that all objects are parts of some other 
things. So, how should we understand a real part? What is acceptable is the view that 'real' means here the same as 'assigned to an object (the whole) rather than actually existing'.

Arguments put forward by Ginsberg have to be presented:

Re 1. It is commonly believed that a relationship is a heteronomous object because of its segments. Nevertheless, for a relationship to exist, existence of its segments is not necessary. Since let us for example consider the following relationship of implication: 'If a tree is a metal, then a tree melts in fire.' States of affairs, marked as antecedent and consequent, are segments of this relationship. Implication relation, that occurs between these two segments, exists resp. occurs, although they themselves do not exist. Similarly, if the contradiction relation occurs between states of affairs, then, perforce, it is always heteronomous towards one existing and one non-existing segment - if the principle of excluded middle and the principle of contradiction are right. If the heteronomous objects do not require the existence of complementary objects, then they do not require existence, which is contemporary with them [3, p. 155].

This reasoning may be questioned in the first instance by an argument, which Ginsberg was aware of. It would be sufficient to assume existence in the broad sense which would make it impossible to acknowledge the existence of objects constituting segments of the relationship discussed. Ginsberg accepts that it is wrong to assign Husserl using this understanding of existence, and even if this would be the case, her criticism could be considered as transcendent towards the Husserl's position.

It is allowed, however, to put forward a more serious objection. It can be reduced to the fact that in the example presented by Ginsberg, a confusion occurred with what Husserl distinguishes and calls formal (analytical) necessities implied from analytical laws a priori, with what is synthetic a priori, thus being foundation for (in)separateness. The theorem that any relationship has segments is an analytical theorem and it may not be a basis for inseparateness.

In addition, serious doubts may be raised towards the term 'state of affairs' used, which has multiple philosophical associations (scholastic aliqualiter, esse, modus se habendi, tantum complexe significable etc. ${ }^{6}$ ) and became a truly technical term, as a result of phenomenologists' work. This was pointed out by Ingarden when he wrote: 'By whom this term (in German Sachverhalt) was introduced into the German philosophical literature, I can not say. Anyway, since the research conducted by phenomenologists - Husserl, Reinach, Pfänder - it has gained the right to citizenship in the philosophical language' [6, p. 178].

This is the interesting because inseparateness is referred to by describing what the very state of affairs is in Ingarden's meaning. At the same time, according to this approach, what is marked by the antecedent is not the state of affairs. The sentence 'A tree is a metal' does not refer to a state of affairs, because analyses of a state of affairs refer to the formal schema: 'a subject of properties properties' and it is in this relationship that we are dealing with inseparateness. Language expresses this by using the subject-predicate sentences like 'this rose here is red' - the state of affairs: the redness of this rose here. For Ingarden, this is the state of qualification of things, distinguished from the states and of appearance of things and the states and of happening of things; the latter would include a consequent. Whether implication (relation) is true or false is based on the true presupposition, which is the state of affairs, 'metal melts in fire'. What occurs here is truth, which means that the content of the state of affairs 'formal object of proposition' overlaps with the 
'material object of proposition'. This distinction means that there can be no relation and nonexistent segments, since both segments and a relation always belong to the same order, regardless of whether they correspond to the objective states of affairs or not. Following Ingarden, it is recommended to say again: 'Each categorical proposition has its own formal object, i.e. an intentional state of affairs presumed in the content of the proposition. If the proposition is true, then, in addition to a formal object, it also has a material object, i.e. an objective state of affairs' [5]. What is also important is that there is no difference in material terms (material content) between a purely intentional state of affairs and an objective state of affairs. The difference between them is of existential nature, the former ones, in contrast to the latter ones, are heteronomous ${ }^{7}$.

In order not to be influenced by simplifications, including for example: denying the antecedent of the implication reference to the state of affairs in Ingarden's meaning, ignoring the peculiarity of the conditional proposition and the absence of an explicit (closely related to inseparateness and separateness) isolation of state of affairs from relation, Ingarden's position should be presented in detail on the issues put forward; I will do it following discussing Husserl's position.

To be more specific about the second Ginsberg's objection towards Husserl's definition of heteronomy, you should keep in mind that in this case, the clarified term 'heteronomy' is equivalent to the Ingarden's term 'inseparateness', having ignored the auxiliary terms ('the whole' and 'parts'), which, as presented here, should be considered as causing difficulties. Let us quote Ginsberg:

Re 2. It is also wrong to claim that a heteronomous object can exist as part of the whole, which - according to Husserl - would be made up along with the object complementary to it. Hence, for example, a property of A being different from B is heteronomous towards both $\mathrm{B}$ as well as A. Nevertheless, a property of being different object A has, does not B make up any whole with object B. Similarly, relations do not make a whole with its segments. They are heteronomous to them, they can only exist as parts of the whole. Thus, for example, a colour to exist requires an inherentive object, of which it could be a part of. However, object's heteronomy, which requires a whole, is just a single type of objects' heteronomy [3, pp. 155-156].

The last sentence of the excerpt quoted expresses the need, which will be satisfied by Ingarden, by distinguishing inseparateness and contingency. Let us however come back to Husserl. This time as well Ginsberg is aware that her criticism is of external nature, because 'being different' belongs to relative properties, whereas Husserl reduces his consideration to absolute properties and does not go beyond the being which is analysed. This example can be refuted in a similar manner as the previous one. Difference of some A and B is not content-grounded but if you say the sentence, 'This redness is different from this greenness', it will be empirical ('this') refinement of synthetic necessity, whereas inseparateness is located within the colourfulness (a multitude of possible concretisations of colours) and the concrete (the uniqueness of the implementation of a specific colour ${ }^{8}$.

This example reveals relativity of (in)separateness: something is (in)separate always in relation to something else. Husserl's definition is as follows:

Inseparate in the whole $G$ and towards it, or towards the set of all contents determined by $G$, is called each of its partial content, which can exist only as part, and only as a part of the whole such species, which is represented in this set. Each 
partial content of which it can not be said, is separate in the whole $\mathrm{G}$ and towards it. In short, we also speak about the inseparate and separate parts of a whole, in the corresponding meaning about inseparate and separate parts of parts (partial wholes) of the whole [4 pt. I, p. 320].

This definition gives rise for at least three important theses.

What we describe as inseparate, may be referred, according to Husserl, to both the whole and to other parts of the whole 9 . Within a single whole, two types of parts were distinguished: separate and inseparate. Reference of the inseparate content to the whole is ambiguous. On the one hand, other contents - parts are in consideration, on the other - the whole is in consideration, which is a summative whole of these contents, and can be of transcendent nature, because it is grounded in the species (type), and due to being the summative whole, it is merely its representation.

This is the right moment to clarify the issues discussed with use of Ingarden's categories. At the point, an obstacle is emerging that was not overcome by Ginsberg. Since she divides the whole into autonomous (separate) and heteronomous (inseparate) parts [Ingarden uses terminology: inseparate-separate, as equivalent], Ginsberg has difficulties in option two (inseparate parts) when she determines certain regularities (parts-wholes). In fact, more precisely speaking, in the first type of this option (inseparate part of and the whole). Types of option one (separate part-separate part, separate part-the whole) overlap with instances occurring within the separate wholes).

Part of the inseparate whole may be inseparate towards: a) the whole it is a part of, b) another part of this whole, c) something (an object) located outside this whole. Regarding the second type, Husserlian Theorem II is important, which is as follows: 'The whole, which contains its part as an inseparate moment, but does not contain the supplement it requires, is inseparate as well, i.e. it is inseparate towards each superior separate whole, which contains this inseparate moment' [4, pt. I, p. 326].

However, using the term 'the whole' twice, in two different meanings, is misleading. The first, inseparate whole is, actually, not a whole. It is desirable to use the term 'unity', while the supplement of, basically, not the part (although, following Twardowski here, a part is extremely broadly understood, 'as a metaphysical part' ${ }^{10}$ as well), but of a moment, is based on founding, and indeed related to the concept of inseparateness as opposed to existential contingency, which occurs between parts in the proper sense, it should be added: between separate parts. This prevents the Ginsberg's error, which is, in turn, highlighted by P. Simons [10], when he says:

Suppose we consider a whole $a$ compounded of the colour and shape (understood as individual accidents, not as universal properties) of a particular brick in wall. This is dependent upon the extension of the brick, and thereby also on the brick itself, but it is not dependent upon the wall, as - according to Theorem II - it should be, a requires the existence of the brick, but not the superordinate wall, since $a$ can perfectly well exist whether the wall does or not, can predate and survive the wall, and would usually do so, as long as the brick did not change in shape or colour.

The criticism and counterexample fail because Ginsberg fails to observe the distinction which Husserl makes between foundation and relative dependence: an individual $a$ of the species a is founded on an individual $b$ of the species $b$ if as have to be supplemented by $b s$ in order to exist at all, and $b$ here does the job for $a$; an individual is dependent relative to another individual if it is founded on something 'within the range of the latter, i.e. is founded on some proper or improper part of it. 
Thus every case of founding is a case of relative dependence, but not vice versa. The brick example is just such a case: the colour \& shape $a$ is founded on the extension of the brick, and the brick is a piece of the wall, so $a$ is dependent relative to the wall, but $a$ is not founded upon the wall. The mistake is quite understandable however, since Husserl is not always consistent in his terms, and introduces them in what, by modern standards, is a sloppy and haphazard fashion.

It can be assumed that Simons, indeed, correctly differentiates relationship of founding and the relative contingency, however the assumption that the latter is contained in the former seems to be less accurate, i.e. all that is founded, it is also relatively contingent, but not vice versa. The situation is different if the Ingarden's order is introduced. Here, what is founded, is existentially inseparate, and what is relatively contingent, is existentially contingent, but everything that is existentially contingent may not also be existentially inseparate, and vice versa. Simons's interpretation involves a presumption that what is linked with the relation of founding, are objects (concretes) ${ }^{11}$. The founding relationship in Husserl's view is rather in species, and it is impossible to think that species are actual parts of objects nor that in this relationship they themselves are parts that make up a whole as the sum of parts. Here is how the situation is described by M. Rosiak:

However, we must not forget what at the same time Husserl says about the nature of elements linked with founding bonds: these are species and types, rather than individuals. It is from this point of view that you should look at an example of connection of two elements, of which one is founded in the other. Elements a can be regarded here as a type, wile ab connections can be regarded as species that falls under this kind. However, this type itself is indeterminate in terms of actual coexistence (or connection) with the moment $b$. An object that falls under type a can exist both as a representative of the species $a b$, and without the determinant $b$. This can be illustrated by using the Aristotelian hierarchy: the type of animal breaks down into two species - rational animal and irrational animal. If we agree that the moment of rationality is founded in animality, then this is not equivalent yet with the claim that these two species are a whole. It is quite obvious that a pair of elements connected with founding relationship does not generate a whole, but two completely different wholes. What kind of the whole will be created depends on whether the moment $b$ is indeed connected with foundation $a$, or whether the latter exists without it [9, pp. 42-43].

This complicated situation is a result of Husserl using terms 'part' and 'whole' in at least two different meanings, and, as highlighted by Simons, is a consequence of assigning the founding relationship to species and individual exemplifications at one go.

These problems become apparent even at the first Husserlian attempt to describe inseparateness:

It can be put more simply by saying: inseparate objects are objects of such [pure] species for which the law [derived from essence] occurs, that if they exist at all, they exist only as parts of larger wholes of a certain [assigned] species. That is the point in the concise expression which says that they are parts that exist only as parts, and they 
can not be conceived as something that exists for itself. The colour of this paper here is its inseparate moment, it is a part of, not only factually, but [according to its essence,] according to its [pure] species is predestined to be a part, because the colour in general [and purely as such] can only exist as a moment in something coloured [4, pt. I, pp. 295-296].

The transition from species to their exemplification is clearly noticeable. Inseparateness can only be described in this double perspective. Its concept comprises 'necessity', thus being founded on relationships of essence rather than actual relationships, but this is not equivalent to the fact that inseparateness does not occur in the realm of actuality. Both species are not parts of actually existing objects (since they have a totally different existential characteristics) and their exemplifications implemented as an object may not be described in this way. They are distinguished by calling them 'metaphysical parts', but 'moment' is a better description, which refers to unity rather than - as in the first case - to the whole that is composed of parts.

When creating a theory of the whole and parts, Husserl was not, however, fully aware of this distinction, although you may detect this intuition emanating in many passages of his considerations. It is more appropriate to admit that until Ingarden nobody accomplished this clearly and completely.

To do justice to things, a detailed analysis of Husserl's position that takes into account the Simons' interpretations is advisable.

\section{Peter Simons' Proposal}

It is hard to reject the general and initial premise of Simons' considerations which states that there are two most important concepts in the Logical Investigations, 'the whole' and 'foundation'. At the same time, they are not defined by Husserl unambiguously ${ }^{12}$. According to Simons, it is recommended to distinguish three types of the whole: narrow, broad and strict, while only the last of these types is the term used by Husserl himself. The wholes in the narrow sense are the wholes which are related with each other with a unifying moment. Many beings create a single whole due to some external thing. For Husserl, this type is not acceptable with respect to all wholes. If it were different way, Husserl would in this respect agree with Twardowski (see page 9). Husserl goes beyond such a concept of the whole, being aware of the danger of infinite regression of parts.

The whole in a broad sense is constituted by being an object. This means that the structure of an object as a whole appears as the most important factor here rather than relationships of its individual parts. In other words, regardless of how strict relationships between the parts are, if each of them can be referred to a specific, single object, they are its parts. You may dare making a statement that the structure of an object is the unifying moment, however, with the important difference that it (an object) is not something external towards its parts. When, however, accuracy of relationships between parts is irrelevant, a hypothesis is acceptable that in this meaning everything is an object (Husserl would have never agreed to this hypothesis because he makes clear contrasts between concept of 'unity' and 'multiplicity'. Another subject of controversy is determining the criterion to distinguish objects with inner unity from those that are wholes in the weakest meaning and either no longer belong to objects (individuals), or still do not belong to them, if we consider a group of individuals as individual in the meaning of the summative whole.

Simons adopts a linguistic criterion, i.e. he holds that all that has proper names in singular is an individual (an object). When defining this type of the whole, Ingarden's distinction should be 
mentioned because what is also useful here is the difference between what is contingent and what is inseparate. The first segment of the division comprises all objects of the higher order as a whole, the second segment comprises an initially individual object. With regard to what is contingent, Ingarden elaborates entire system of possible types of relationships between parts of such wholes, which will be discussed elsewhere. On the other hand, the second Ingarden's option corresponds to what is here referred to as a strict whole, i.e. the whole whose parts are in themselves directly or indirectly founded and nothing that constitutes those parts is founded in anything except the whole. Nothing stands in the way, in this context, of the term 'funded' being regarded as interchangeable with the term 'inseparate', as Husserl himself does [4, pt. I, p. 326]. The inner unity of 'parts' (moments) in such a whole is inseparable. Unlike the summative whole, it is not founded on an external moment of unity, since in all wholes contradictory to this unity, parts of these wholes are separate. Husserl himself has perfectly expressed it when he wrote:

Anyway what strikes us is the fact that, where the occurrence of connecting forms may indeed be stated as [individual moments of intuition], connected parts are separate towards each other, e.g. sounds in the unity of a melody or isolated pieces of colouring in unity of colour configuration or partial figures in unity of some more complex figure, etc. Whereas our efforts are in vain, when in visual unity of the phenomenon, apart from contents constituting forms that unify pieces, we want to locate also those that combine with each other [inseparate moments] e.g. colour and extension or that combine hue and brightness within the colour, while [they combine the moment of form with the moment of size] within extension, etc. [4, p. 346].

Of course one might put forward objection that being influenced by this paragraph, what is described here is merely relationships limited to certain content, rather than objects, so this would be true only in relation to the realm of phenomenology, and the state of affairs described here could result from some deficiencies within the theory of cognition. Husserl anticipated this type of objection and adopted the relationship of founding as a fundamental relationship for all the wholes considered:

Obviously, this approach [applies not only to the realm of intuitive objects (in particular phenomenological content) which we used as an example, but to the realm] of objects in general. We are even inclined to say: what really unifies are the relationships of founding. As a result, unity of separate objects is created by founding as well. Since they are not founded one in another, because they are separate, what remains is the possibility that they themselves, even jointly, are foundation for the new contents, which, because of the situation with regard to the founding 'segments', are called unifying contents [4, p. 348].

We quoted quite extensive excerpts of Logical Investigations because they comprise in full Husserl's discussion of types of the whole, showing not only how they kind of intersect each other, but also how they differ. First of all, our intention is to highlight the difference in the relationship of parts of the wholes defined, i.e. inseparate and separate "parts". In the first case, words of Husserl are symptomatic of this whole, and more specifically: of this unity, which once again are worth quoting: 
But those we refer to, have a lot in common, they are after all founded one in another and that is why they need no chains or ties [...], to gather them together. Actually, all these expressions do not have any sense in their case. Where isolating them makes no sense, there the problem of how to overcome this isolation makes no sense as well [4].

Moments in the relationship of inseparateness, which are called here inseparate parts, may not be isolated. They themselves form a unity by means of the relationship, intersecting each other, and this unity is something else than the unity of the summative whole, and alternatively of other wholes united by the moment of unity. In both these cases isolation of parts can be done and is carried out in the same way as respectively in collective and distributive sets. Intersection of moments of unity, which is the essence of inseparateness, excludes this type of operation of isolating these moments and constitutes peculiarity of this type of structure. This peculiarity is manifested in the unity of inseparate moments. This situation is vividly illustrated by Rosiak:

Whereas, when we take the coloured extension as an example and assume that the extension is the foundation of colourfulness (but not vice versa), no one will expect that it will be possible to divide this whole, so that a colourless extension appears as a correlate with the abstract colouring since it is not a part of the whole we consider. If it was the case, this colour would be a sort of crust put on it rather than the moment that intersects with it [9].

\section{Final thoughts}

At first, the fact that the Husserl and Ingarden derive definitions of these terms from the part and the whole in a different way, was the basic difference within the theory of inseparateness/separateness between Husserl and Ingarden.

Husserl defines both terms, referring parts to the whole. One time, the whole is made up of an object and its parts, which are perceived as separate or inseparate (moments of properties), another time he refers parts of the given whole to parts of another whole on the background of species and types [Theorem II].

Whereas Ingarden describes inseparateness as a 'mutual' relationship of "parts" (moments) within the whole (unity), while pointing out at the same time that either this is (inseparate) unity, i.e. once again referred to some kind of the whole or separate and it no longer is inseparate towards anything. At the level of species and types, Ingarden does not relate them to each other as (separate) wholes, because there is no need to do so, although these types of areas are elaborated based on corresponding distinctions.

The example given by Rosiak can be as well replaced by unilateral inseparateness of types towards species.

What is common for Husserl and Ingarden, however, is the belief that inseparatenesses are primarily derived from matter without taking account of form. When the primary types of inseparateness will be discussed (formal and material inseparateness), it will turn out that for Ingarden this is a more complicated issue.

\section{References}

1. Biłat, A. Truth and the States of Affairs [in:] A. Biłat (Ed.). States of Affairs, Situations, 
Events. Vol. I. Lublin: Publishing House of University of Maria Curie-Sklodowska, 1995.

2. Borkowski, L. Letters on Truth, and the States of Affairs, [in:] A. Biłat (Ed.). States of Affairs, Situations, Events. Vol. I. Lublin: Publishing House of University of Maria CurieSklodowska, 1995.

3. Ginsberg, E. Concerning the Concepts of Inseparateness and Separateness. [in:] Memorial Book of the Polish Philosophical Society in Lviv 12.2.1904-12.2, Lwów 1929, s. $143-168$.

4. Husserl, E. Logical Investigations, Vol. II, transl. by J. Sidorek. Warszawa: PWN, 2000.

5. Ingarden, R. At the Foundations of the Theory of Knowledge. Warszawa: PWN, 1971.

6. Ingarden, R. On Literary Works, transl. by M. Turowicz. Warszawa: PWN, 1960.

7. Łaciak, P. Kant and Husserl and the Problem of the Material a priori, Principia, vol. XXXV-XXXVI. Kraków 2003-2004.

8. Laciak, P. The Structure and Types of Cognition a Priori As Understood By Kant and Husserl. UŚ Katowice, 2003.

9. Rosiak, M. Formalising Ontology of Founding, Philosophy of Science, IV, 1996, No. 1 (13), p. 45 .

10. Simons, P. On the Concepts of Existential Dependence and Independentce (elaborated by E. Ginsberg) [in:] Parts and Moments Studies in Logic and Formal Ontology, red. B. Smith, Analytica, München 1982, p. $261-282$.

11. Smith, B. Sachverhalt, [in:] J. Ritter, K. Gründer (Eds.). Historisches Wörterbuch der Philosophie, Vol. VIII. Basel/Darmstadt: Schwabe \& Co Verlag, 1992.

\section{Notes}

1. Ingarden's innovation, which consists in classifying these moments as existential moments, is also important. Some researchers question its legitimacy. This is however a separate issue that requires a more advanced reasoning.

2. What I mean here are also all these instances, typical of English-speaking commentators in the subject we discuss, who intend to qualify the ontological inseparateness within the ontological incontingency, but at the same time they make distinction between the Husserlian foundation and relative dependence (Peter M. Simons). This position is inconsistent with the Ingarden's suggestion. At the same time, when interpreting the Ingarden's ontological moments, the difficulty also occurs at the level of the language itself because following defining the four pairs of ontological moments, elaborated by Ingarden, including the ontological contingency, you come across the fifth one, comprising all of them and referred to as'dependence'. Cf. A. L. Thomasson, Ingarden and the Theory of Dependence, translated by Artur Mordka, 'Sofia', no. 3/2003, UR.

3. Therefore, predicating on the very existential moments as existentially inseparate in relation to the mode of being as a whole, is unacceptable.

4. 'Abstraction in the meaning of the very act is something completely different from the mere paying attention to a moment of being red or highlighting it, to mark this difference we talked about the ideating or generalising abstraction in multiple instances. This very act is what the traditional way of talking about abstraction refers to, according to it, what we get with "abstraction" are not particular individual features, but the general concepts (direct presentations of attributes as unities in thinking). Sometimes this manner of speaking is extended to the conceptual presentations of the complex forms mentioned, the presentation of some A, a lot of A etc. All other properties are disregarded, the abstract presentation of A adopts new "forma", but no new "matters" [4, pt. I, p. 274].

5. E. Ginsberg uses terms 'inseparate/separate' in the meaning in which Ingarden uses 'heteronomous/autonomous' in his terminology.

6. See [2, p. 2]. Also [11].

7. This was pointed out well as by [1, p. 99].

8. '[Thus, the concept of inseparateness is equivalent to the concept of the ideal] being subordinate to the law in uniform relationships. If a part is a part of not just factual relationship but determined by the [ideal] law, then it is inseparate, because this relationship determined by the law does not mean anything but a the fact that a part that belongs to such species [in accordance with its pure essence] under the law it can only exist as combined with some other parts, which belong to these or those species assigned. Also, where the law regulations apply to the impossibility instead of necessity of a combination, for example, where it states that the existence of part A excludes the existence of part B as contradictory to it, we reach inseparateness in there as well. Since A may 
exclude B only so that they both demand the same in a mutually exclusive way. One colour excludes a different colour - but on the same piece of surface that they both would completely cover, although they both can not do this' [4, pt. I, p. 309-310]. The above excerpt also prompts to add a general comment, i.e.: this type of approach can not be interpreted extensionally and the Husserlian theory of the whole and the part should be accepted only along with the Platonic experience and associations. Also P.M. Simons, Three Essays in Formal Ontology, refers to this aspect [in:] Parts and Moments Studies in Logic and Formal Ontology, Analytica, München 1982, p. 116.

9. See Ginsberg's remarks regarding the understanding of inseparateness by Twardowski.

10. To distinguish between two types of parts, also the term 'elements' is used (even by Twardowski). This issue is also discussed by Marek Rosiak in the article [9], when he wrote: 'If, therefore, some elements of the whole do not make up parts of it, then does it make sense to talk about compliance with Theorems that Husserl states on this issue, and in particular with his view that all that can be distinguished in an object is a part? If parts in the strict sense were the only type of parts, then, of course, compliance does not make any sense. But in the second part of Logical Investigations Husserl speaks in favour of the fact that there are no other parts as the type just mentioned. These two theorems may not be held consistent at the same time. A generic term must also be taken into account as part of an object, or it must be concluded that not everything that is related to each other by founding relationships in an object is a part of it' [9, p. 45].

11. In terminology used by Ingarden: object in the strict sense, which is the subject of properties. Properties are regarded here as objects.

12. The conjecture that the absence of formalisation of the theory of the whole and the part is Husserl's deliberate act, should also be accepted. It is worth mentioning that such action was motivated, in particular, by the fact that the founding relationship and inseparateness relationship, which is of no small importance in this theory, are related with material laws and as such they are not subject to formalisation. 